\title{
Open Problems
}

\section{compiled by Kefeng Liu*}

Readers are invited to propose solutions to the following problems. Solutions should be sent to liu@math.ucla.edu, and posted on ArXiv. The correct solutions will be announced, and tokens of appreciation will be presented to the solvers.-the Editors.

Problem 2014002 (Differential Geometry). Proposed by Shing-Tung Yau, Harvard University, USA.

Prove that every compact Riemannian manifold with zero Ricci curvature admits a stable closed geodesic.

Problem 2014003 (Differential Geometry). Proposed by Feng Luo, Rutgers University and Shing-Tung Yau, Harvard University.

Suppose $X$ and $Y$ are two strictly convex smooth closed surfaces in $\mathbb{R}^{3}$ and $F: X \rightarrow Y$ is a diffeomorphism preserving the second fundamental form. Is $F$ an isometry?

The best work is by V. Grove [1] in 1957 who proved that if in addition $F$ preserves the Gaussian curvature, then $F$ is an isometry. Vortisch and Walden [2] in 1970 proved that if in addition $F$ preserves the principle curvature, then $F$ is an isometry. This question is motivated by the Stoker's rigidity conjecture on convex compact polytopes.

We can also ask a generalization of it in higher dimension:

Suppose $X$ and $Y$ are two strictly convex compact hypersurfaces in $\mathbb{R}^{n}$ for $n>2$ and $F: X \rightarrow Y$ is a diffeomorphism preserving the second fundamental form. Is $F$ an isometry?

[1] Grove, V. G. On closed convex surfaces. Proc. Amer. Math. Soc. 8 (1957), 777-786.

\footnotetext{
* Department of Mathematics, University of California, Los
} Angeles

E-mail: liu@math.ucla.edu
[2] Vortisch, W., Walden, R. Ein Kongruenzsatz für Eiflähen. (German) Manuscripta Math. 3 (1970), 315-319.

Problem 2014004 (Néron-Ogg-Shafarevich for K3 surfaces, Arithmetic geometry). Proposed by ChingLi Chai, Academia Sinica, Taipei/ University of Pennsylvania, USA.

Let $R$ be a complete discrete valuation ring with residue field $\overline{\mathbb{F}}_{p}$ and fraction field $K$. Let $X$ be a (smooth proper) K3 surface over $K$. Suppose that $\operatorname{Gal}(\bar{K} / K)$ operates trivially on $\mathrm{H}_{e t}^{2}\left(X \times_{\operatorname{Spec}(K)} \operatorname{Spec}(\bar{K})\right.$. Does there exist a proper smooth scheme over $R$ whose generic fiber is isomorphic to $X$ ?

Remark. The analogous statement is true if $R \cong F[[t]]$ for an algebraically closed field $F \supset \mathbb{Q}$.

Problem 2014005 (Nonlinear Elliptic PDEs). Proposed by Jun-cheng Wei, The Chinese University of Hong Kong, China.

Consider the following system of elliptic equations modeling Bose-Einstein condensates

$$
\left\{\begin{array}{lll}
\Delta u-u+u^{3}+\beta u v^{2}=0 & \text { in } & \mathbb{R}^{N} \\
\Delta v-v+v^{3}+\beta v u^{2}=0 & \text { in } & \mathbb{R}^{N} \\
u, v>0, u, v \in H^{1}\left(\mathbb{R}^{N}\right) & &
\end{array}\right.
$$

where $N=2,3$. By the method of moving plane, all solutions are radially symmetric so we may restrict in the class of radial functions. It is known that there is the following type of solutions

$$
(u, v)=\left(c_{1} w, c_{2} w\right), c_{1}>0, c_{2}>0
$$

where $w$ is the unique radial solution of the single equation $\Delta w-w+w^{3}=0$.

Are all solutions to (1) given by (2)? 
Remark. It is known that this is true when $\beta \geq 1$. So we may assume that $\beta \in(0,1)$.

Problem 2014006 (Differential Geometry). Proposed by Qing-Ming Cheng, Fukuoka University, Japan.

Prove that an $n$-dimensional complete selfshrinker $X: M^{n} \rightarrow \mathbb{R}^{n+1}$ in $\mathbb{R}^{n+1}$ has a polynomial volume growth.

Remark. If one assumes that an $n$-dimensional complete self-shrinker is proper, the result holds. See Q. Ding and Y. L. Xin (arXiv:1101.1411, 2011) and X. Cheng and D. Zhou (arXiv:1106.4950, 2011).

Problem 2014007 (Complex Geometry). Proposed by Shing-Tung Yau, Harvard University, USA.

Formulate an analogue of Hodge conjecture for symplectic manifolds. For a Calabi-Yau manifold with dimension $n$, is every $n$ dimensional homology class (over real number) representable by special lagrangian cycles? Can one realize it in a way that is compatible with the mirror map as is described by the SYZ construction. Note that the mirror image of a complex cycle could also be a coisotripic A-brane.

Remark by Conan Leung. Near the large complex structure limit, there should not be any extra Hodge cycle, thus lagrangian cycles should be enough to captured mirror of complex cycles. However, going into the interior of the moduli space of complex struc- tures (in dimensions at least four), there should be places where extra Hodge classes appear. I expect that the mirror of these extra Hodge classes should be (special) co-isotropic A-branes. Some evidences for Abelian surfaces are given by Kapustin-Orlov.

Problem 2014008 (Complex Geometry). Proposed by Shing-Tung Yau, Harvard University, USA.

For a Calabi-Yau fourfold, we look at those homology classes that can be represented as a combination of Cayley cycles: those that calibrated by the sum of the real part of a holomorphic 4-form and half of the square of the Kähler form. Will they be mapped to homology classes of similar type under the mirror map?

Problem 2014009 (Graph Theory). Proposed by Shing-Tung Yau, Harvard University, USA.

Given a group, and a generating set within it, one can define a graph. It is called a Cayley graph. But the structure of the graph depends on the generating set. Given two different generating sets, how do we determine whether the graphs are isomorphic or not isomorphic. What are the graph invariants that can be determined which are independent of the generating set. Find sufficient conditions for a discrete graph to be a Cayley graph, other than the Sabidussi Theorem: A graph is a Cayley graph of a group $G$ if and only if it admits a free and transitive action of $G$ by graph automorphisms. 